\title{
Knowledge sharing between general and pediatric emergency departments: connections, barriers, and opportunities
}

\author{
Leah K. Crockett, BHSc, MSc**; Carly Leggett, BKin, BEd, $\mathrm{MPH}^{*{ }^{\dagger}}$; Janet A. Curran, RN, MEd(BN), PhD ; \\ Lisa Knisley, BN, MA ${ }^{\dagger}$; Gwenyth Brockman, BSc*; Shannon D. Scott, RN, PhD ; Lisa Hartling, PhD ${ }^{\text {III; }}$ \\ Mona Jabbour, MD, MEd ${ }^{* *^{\dagger+}}$; Terry P. Klassen, MD, MSc ${ }^{{ }^{\dagger \ddagger \ddagger}}$
}

\begin{abstract}
CLINICIAN'S CAPSULE
What is known about this topic?

Evidence-based pediatric emergency care is variably applied across general and pediatric emergency departments.

What did this study ask?

What are the information-seeking patterns of pediatric emergency medicine network members?

What did this study find?

There remains a need to share successful methods of local dissemination and implementation across the network, and to leverage local professional champions such as clinical nurse liaisons.

Why does this study matter to clinicians?

Tailoring knowledge mobilization efforts to end-user priorities is essential to ensure impact and spread of outreach efforts.
\end{abstract}

\section{ABSTRACT}

Objective: Translating Emergency Knowledge for Kids (TREKK) is a national network aimed at improving emergency care for children by increasing collaborations and knowledge sharing between general and pediatric emergency departments (EDs). This study aimed to determine patterns of knowledge sharing within the network and to identify connections, barriers, and opportunities to obtaining pediatric information and training.

Methods: We conducted 22 semi-structured interviews with health care professionals working in general EDs, purposefully sampled to represent connected and disconnected sites, based on two previous internal quantitative social network analyses (SNA). Data were analyzed by two independent reviewers.

Results: Participants included physicians (59\%) and nurses (41\%) from 18 general EDs in urban (68\%) and rural/remote
(32\%) Canada. Health care professionals sought information both formally and informally, by using guidelines, talking to colleagues, and attending pediatric related training sessions. Network structure and processes were found to increase connections, support practice change, and promote standards of care. Participants identified personal, organizational and system level barriers to information and skill acquisition, including resources and personal costs, geography, dissemination, and time. Providing easy access to information at the point of care was promoted through enhancing content visibility and by embedding resources into local systems. There remains a need to share successful methods of local dissemination and implementation across the network, and to leverage local professional champions such as clinical nurse liaisons.

Conclusions: These findings reinforce the critical role of ongoing network evaluation to improve the design and delivery of knowledge mobilization initiatives.

\section{RÉSUMÉ}

Objectif: Translating Emergency Knowledge for Kids (TREKK) est un réseau national qui vise à améliorer les soins d'urgence aux enfants en favorisant la collaboration entre les services des urgences générales (SUG) et les services des urgences pédiatriques ainsi que la mise en commun des connaissances. L'étude avait donc pour buts, d'une part, de déterminer les différentes formes de mise en commun des connaissances au sein du réseau et, d'autre part, d'examiner certains aspects liés à l'obtention de l'information en pédiatrie et à la formation, soit les relations, les obstacles et les possibilités.

Méthodes: Vingt-deux entretiens semi-dirigés ont été réalisés avec des professionnels de la santé travaillant dans des SUG et échantillonnés à dessein afin que soient représentés des services des urgences en relation ou non avec des établissements-ressources, d'après des données internes et quantitatives,

From the ${ }^{*}$ George \& Fay Yee Centre for Health Care Innovation, Winnipeg, MB; †Children's Hospital Research Institute of Manitoba, Winnipeg, MB; $\neq$ School of Nursing, Dalhousie University, Halifax, NS; §Faculty of Nursing, University of Alberta, Edmonton, AB; ๆDepartment of Pediatrics, University of Alberta, Edmonton, $A B$; $\| A$ lberta Research Centre for Health Evidence, University of Alberta, Edmonton, $A B$; ${ }^{*}$ Department of Pediatrics, University of Ottawa, Ottawa, ON; ††Department of Emergency Medicine, University of Ottawa, Ottawa, ON; and the $¥ \ddagger D e p a r t m e n t ~ o f$ Pediatrics and Child Health, University of Manitoba, Winnipeg, MB.

Correspondence to: Leah Crockett, 374-1 753 McDermot Avenue, Winnipeg MB R3E 0T6, Canada; Email: Icrockett@hsc.mb.ca 
recueillies dans deux analyses de réseaux sociaux menées antérieurement. Les données ont été analysées par deux examinateurs indépendants.

Résultats: Ont participé à l'étude des médecins (59\%) et des infirmières ( $41 \%$ ) provenant de 18 SUG situés en milieu urbain ( $68 \%$ ) ou encore en milieu rural ou en région éloignée (32\%), au Canada. La recherche d'information par les professionnels de la santé se faisait de manière tant structurée que non structurée, par exemple la consultation de lignes directrices, les échanges de points de vue avec des collèges, la participation à des séances de formation en pédiatrie. II s'est révélé que la structure du réseau et ses processus amélioraient les relations, soutenaient les changements de pratique et favorisaient I'application des normes de soins. Les participants ont soulevé des obstacles personnels, organisationnels et systémiques à l'acquisition de l'information et de compétences, notamment le coût des ressources et les coûts personnels, la distance, la diffusion de l'information et le manque de temps. L'obtention de I'information au point de prestation des soins a été facilitée par une amélioration de la visibilité du contenu et par l'intégration des ressources dans les systèmes locaux. II reste toutefois à trouver des méthodes efficaces de diffusion locale de l'information et d'application des connaissances dans tout le réseau ainsi qu'à former des guides professionnels à l'échelle locale comme les infirmières cliniciennes de liaison.

Conclusions: Les résultats de l'étude viennent renforcer le rôle crucial de l'évaluation continue du réseau afin d'améliorer la conception et la réalisation d'initiatives de mobilisation des connaissances.

Keywords: barriers, emergency medicine, facilitators, knowledge mobilization, information sharing, pediatrics

\section{INTRODUCTION}

Use of the latest evidence is key to the delivery of highquality care. In the field of emergency medicine, the scope of evidence is vast, and there is a well-cited disconnect between knowledge production and knowledge use. ${ }^{1}$ The emergency department (ED) environment presents a number of unique challenges to sustained knowledge acquisition and sharing. ${ }^{2}$ Further challenges exist in community EDs where the staff is trained to meet the needs of the general population, are exposed to fewer pediatric cases, and have limited access to current and reliable resources, thus restricting exposure to new information and knowledge. The mixed-staffing model in many rural EDs, where intermittent coverage is provided by family physicians and other specialists, presents further challenges in knowledge sharing and upkeep. ${ }^{3}$ In Canada, $85 \%$ of children seek care outside of pediatric centres where the vast majority of knowledge is produced. ${ }^{4}$ It is estimated that as many as $40 \%$ do not receive treatments for which clear evidence exists, and one in five may receive treatments that are of no benefit or could even be harmful, ${ }^{5-8}$ indicating a pervasive gap in the translation of the latest knowledge into practice.

Translating Emergency Knowledge for Kids (TREKK), a national network in pediatric emergency care, was established in 2011 to address these critical knowledge-to-practice gaps and accelerate the speed with which the latest evidence is shared and used in nonpediatric EDs. The TREKK network includes partnerships with 37 general EDs spanning nine provinces and one territory. Known as TREKK sites, each of these 37 institutions is partnered with its closest Pediatric Emergency Research Canada (PERC) site, within teaching or pediatric hospitals. The potential that networks have to address complex challenges is increasingly accepted in multiple disciplines. ${ }^{9-11}$ Health care professionals rely heavily on their social networks to find information and make clinical judgments. ${ }^{2,12-14}$ Advantages of network coordination include enhanced learning, more efficient sharing and use of resources, increased capacity to address complex issues, reduced duplication, and rapid diffusion and reach ${ }^{10,15}$; however, measuring these impacts can be challenging. Social network analysis (SNA) uses network theory to understand patterns of interaction ${ }^{16}$ and presents a unique, but underused, opportunity to complement quantitative techniques with qualitative methods to gain insight into both network structures and processes. ${ }^{17,18}$

The purpose of this study was to build on the results of our two previous SNA measures to gain a more in-depth understanding of the network processes and context. The previous SNA measures provided important information on the overall structure of the TREKK network, including locating the knowledge brokers of the network and identifying a reduction in the number of disconnected sites between 2014 and 2015. ${ }^{19}$ Disconnected EDs were defined as those not connecting with a pediatric centre or other health institution for information seeking or advice. The specific aims of this qualitative study were to 1) determine patterns of knowledge sharing and connectivity among rural, urban, and community EDs; and 2) identify strategies and initiatives used to share knowledge in pediatric emergency medicine, including current barriers and opportunities. 


\section{METHODS}

\section{Sample}

All health care professionals working in the EDs of TREKK sites were eligible to participate in this study. Sites were purposefully sampled to ensure representation from across Canada, with the aim of conducting at minimum two interviews per province. Informed by the results of previous quantitative SNAs conducted in 2014 and 2015, the sampling technique aimed to achieve representation from both connected and disconnected sites. Staff working in general EDs were invited to participate through their TREKK site representatives, who are senior clinicians and managers of their hospital sites and linking agents to the TREKK network.

\section{Data collection}

An interview guide was developed by the study team and piloted with three health care professionals (see Appendix). Data were collected through 22 individual semi-structured telephone interviews with health care professionals working in TREKK-affiliated general EDs between September and November 2016 ranging from 5 to 20 minutes in duration. Interviews were audio-recorded and were transcribed intelligent verbatim by an independent company. The interviewer reviewed transcripts to ensure accuracy and to remove any identifying information.

\section{Data analysis}

Interviews were analyzed by two members of the research team using content analysis. ${ }^{20}$ Each reviewer read through all interviews and independently open-coded $25 \%$ of the interview transcripts. Reviewers then compared and contrasted codes until an inter-coder agreement of $>0.80$ was achieved. Initial codes were established, and the remaining data were coded by both reviewers and managed using QDA Miner software. The reviewers employed analyst triangulation with the study team to develop themes and to ensure credibility and inter-coder agreement throughout the remaining analysis.

\section{Ethical considerations}

Approval was obtained from the University of Manitoba Health Research Ethics board (\#H2016:249). Informed consent was obtained from all participants before commencing the interviews. Randomly generated codes were assigned to each name and location to protect participants' privacy. Links between the name and code number are housed on a password-protected file and computer at the Children's Hospital Research Institute of Manitoba.

\section{RESULTS}

The study population consisted of physicians (59\%) and nurses (41\%), representing 18 non-pediatric EDs. Participating ED sites were situated in both urban (68\%) and rural/remote (32\%) areas spanning 10 provinces and one territory of Canada. Slightly more males $(\mathrm{n}=12)$ than females $(\mathrm{n}=10)$ participated. Physician participants were more likely to be male $(85 \%)$, and nurse participants were more likely to be female (91\%). Participating sites were located between 2 and $1500 \mathrm{~km}$ from their nearest PERC site (average $215.94 \mathrm{~km}$; standard deviation $[\mathrm{SD}]=364.56$ ).

Major themes emerging from the analysis included: 1) patterns of knowledge acquisition and sharing; 2) barriers to obtaining pediatric information, training, or both; 3) opportunities to enhance capacity; and 4) benefits of affiliation with the TREKK network.

\section{Theme 1 - Patterns of knowledge acquisition and sharing}

Health care professionals working in general EDs reported using a range of pediatric information sources including guidelines, online resources, and colleagues and various means of accessing the information, including seeking advice from local or offsite pediatric experts, and attending pediatric-specific professional development opportunities. Guidelines and other online resources were frequent sources of information for both nurses and physicians. As a second line of information, participants reported talking to colleagues within their ED and local pediatricians. One of the initial objectives of TREKK was to increase connections between general and pediatric EDs. Contacting the nearest PERC site for information was consistently noted across all participants, particularly for unique cases or if uncertainty remained after consulting the latest guidelines. The PERC site was also used as a resource in instances in which health care professionals felt their colleagues' practices were not in line with the latest evidence (Box 1; nurse 2109-MQ). Physicians 


\begin{tabular}{|c|c|}
\hline Key findings & Quotes \\
\hline Culture \& connections & $\begin{array}{l}\text { - "Pediatrics works great; they sort of reassess and help us. It's advantageous in the rural area, where we're just not } \\
\text { quite sure ... they're very good at supporting us." (physician 2123-YA) } \\
\text { - "The PERC site has always been known to be very helpful, so it's never been a struggle for us. I think that culture } \\
\text { has definitely helped us feel more open to contacting them and also that we didn't really need any other resources, } \\
\text { because it's such a great resource to have." (physician 2422-XA) } \\
\text { - "I mean the information is out there; most people know how and where to find it. And if you're really, really stuck, } \\
\text { sometimes we would then call the PERC site." (physician 2275-JY) } \\
\text { - "If it's something that came from TREKK, concussion, gastro, anything like that . . we have that information, and } \\
\text { that's what we'll go by. If we have individual questions, so, for example, we have a physician who just can't give up } \\
\text { on [x practice] ... so when that happens, we phone the PERC site and get their most up-to-date information on } \\
\text { [x practice], and that's what we'll go by." (nurse 2109-MQ) }\end{array}$ \\
\hline Comfort levels & $\begin{array}{l}\text { - "It's getting people to be comfortable dealing with pediatric cases. So, seeing as much as we can, sharing } \\
\text { knowledge, these things help." (nurse } 1270-\mathrm{PI} \text { ) } \\
\text { - "They come out of nursing quite comfortable with their adult knowledge, but when I ask what area they want to } \\
\text { improve in their practice, invariably, they say pediatrics." (nurse } 2109-\mathrm{MQ} \text { ) } \\
\text { - "We don't see a lot of sick children or infants. And when we do, it's always very . . . oh, you know, no one is } \\
\text { comfortable." (physician } 2220-J Y \text { ) } \\
\text { - "I think there's a change in perspective required. It's really only when these nurses experience a truly critically ill } \\
\text { child that they are almost scared into seeking education beyond what they've already received." (nurse 2021-Ol) }\end{array}$ \\
\hline Barriers & $\begin{array}{l}\text { - "It's just the unfamiliarity of kids, so I think that comes with us probably not seeing enough sick, sick kids, you know } \\
\text {... to be totally comfortable." (physician } 2275-J Y \text { ) } \\
\text { "The government will not give us a pediatric floor even though we have an increasing number of pediatric visits and } \\
\text { serious cases. We have to transfer out more children than our two hospital corporation sites combined, and that's } \\
\text { not always optimal for the patient." (physician 2025-LQ) } \\
\text { - "I can think of at least 8-10 hospitals in the city that don't have the services of a pediatrician. For those hospitals, it's } \\
\text { important to have maybe a bit more direct channels or protocols in place to facilitate the transfer of kids." (physician } \\
\text { 1556-AV) }\end{array}$ \\
\hline $\begin{array}{l}\text { Supporting practice } \\
\text { change }\end{array}$ & $\begin{array}{l}\text { - "We actually used one of your bottom line recommendations. . . We had a thing going on with ortho follow-up and } \\
\text { casting; people just had to stop referring. But it can be hard for people to change practices they've been doing for } \\
10 \text { years, you know. Having it in writing from an organization [that] has synthesized the information allowed us to } \\
\text { say, look... you're not going out on a limb here, you're well supported by this organization. And that made them } \\
\text { comfortable to change, very much so." (physician } 2155-B X \text { ) } \\
\text { " "It's important to understand how important TREKK is to us and how it's being integrated at the jurisdictional level. } \\
\text { So, it's working. And I think that we'll be a good example of how TREKK is really changing practice." (physician } \\
2450-\text { CR) }\end{array}$ \\
\hline $\begin{array}{l}\text { Promoting standard } \\
\text { of care }\end{array}$ & $\begin{array}{l}\text { - "TREKK has been a godsend actually, in sort of clarifying some of this stuff and getting us all on the same page ... } \\
\text { the pediatricians, emergency physicians, and family doctors. Especially those one-pagers." (physician } 2475-K C \text { ) } \\
\text { - "TREKK is closing that whole knowledge translation gap ... helping to promote standard of care and share best } \\
\text { practices. So that whole idea that if you presented with status epilepticus or asthma in a PERC site or general ED, } \\
\text { that you would still get equal treatment." (physician 2155-BX) } \\
\text { - "It's comforting to know that what we're doing for say, asthma, is standard of care at the PERC site... so that kids } \\
\text { are getting the same treatment." (physician 2450-CR) }\end{array}$ \\
\hline
\end{tabular}

reported engaging in more formalized methods of information seeking, including attending rounds or journal clubs and through telephone consults. Physicians working in rural areas described the overall culture in pediatrics as one that facilitated access to information (physician 2123-YA; 2422-XA). Alternatively, physicians in urban sites were more likely to report contacting the PERC site as a later step in the information-seeking process (physician 2275-JY). Nurses reported using more informal methods to seek information, such as talking to local physicians or contacting nurse educators at their nearest PERC site for the most up-to-date information.

Achieving an adequate level of comfort with pediatric cases was critical for both physicians and nurses (nurse 1270-PI; 2109-MQ; physician 2220-JY). Professional 
development activities were highly valued across all demographics, serving to increase pediatric knowledge and comfort levels. Hands-on training through simulation scenarios, outreach education from the nearest PERC site, and sharing learnings with colleagues were the most frequently mentioned methods to increase comfort. Notably, an uptake in training often occurred following a critical or "scary" event (nurse 2021-QI).

\section{Theme 2 - Barriers to obtaining pediatric information, training, or both}

Participants reported organizational, personal, and system-level barriers to achieve the desired level of pediatric information and training. Barriers at the organizational level included challenges disseminating resources to staff, geographical barriers relating to patient transfers and attending professional development sessions, organizational resources (e.g., funding for professional development and staffing levels), low staff interest, and infrequent exposure to training opportunities. Participants reported personal barriers including time (e.g., competing clinical responsibilities), personal costs (e.g., paying out of pocket or using vacation days for training), and a low volume of children seen at their site (physician 2275-JY). At the systems level, policy level decisions were sometimes at odds with staff views and resulted in situations that were not always viewed as optimal for a patient (physician 2025-LQ; 1556-AV).

\section{Theme 3 - Opportunities to enhance capacity}

At the site level, enhancing the visibility of pediatric resources (e.g., through TREKK desktop icons) and having easy access to online pediatric protocols and resources facilitated information use. Pediatric liaison roles were key to the organization and provision of pediatric information and training, and, if absent, an inherent barrier. Reducing personal costs by facilitating reimbursement through nursing unions or recruitment and retention funds were noted as ways to enhance training attendance. At the systems level, embedding guidelines into local care systems, such as pediatric order sets and regional care maps and protocols, facilitated the use of best practices. Developing relationships early on, by promoting TREKK in medical school or by building sessions at the nearest pediatric site into orientation training, provided lasting connections to the children's hospital and an instant link to an information source for pediatric emergency medicine. Of note, this strategy would be challenging for sites in rural and remote areas of Canada.

Participants identified opportunities to facilitate the dissemination and implementation of best evidence. Disseminating information to staff was often a challenge, suggesting a need to share successful methods of dissemination and implementation between TREKK sites. Nurses reported not having enough time to look online for information while delivering care, particularly in busy emergency settings. Embedding resources into local systems and providing easy access to resources at the point of care were suggested strategies to reduce barriers to information use. Some physicians reported using pediatric order sets for available conditions but noted a lack of them, especially for less common, more anxiety-provoking cases such as seizures. One physician suggested replicating successful initiatives from the adult field, including ongoing monitoring and evaluation of patient outcomes, transfer outcomes, and comparability to peer hospitals. Furthermore, one participant spoke of the need for better-developed protocols between institutions at the provincial or national level, particularly relating to criteria for transport.

Nurses often reported a "pull" (having to actively seek out needed information) versus "push" (tailored dissemination of knowledge and resources to those who need it) methodology for acquiring information, and both nurses and physicians expressed a desire for central coordination and promotion of events and practice changes. A prominent theme that emerged was the essential role of clinical nurse educators to facilitate training opportunities, as well as their potential to transform current efforts from a pull to a push methodology and to enhance connections between pediatric and general ED sites. In particular, nurse participants identified a shortage of available training opportunities and different training standards for those working within and outside of pediatric centres. Standardizing training requirements and reducing financial barriers to obtaining training in pediatrics was thought to be an essential step forward. "More contact, more simulation, more outreach education" was a common sentiment, suggesting that for comfort levels to increase, more frequent exposure is necessitated through the maintenance and enhancement of network connections. 


\section{Theme 4 - Benefits of affiliation with the TREKK network}

Participants described numerous benefits of affiliation with the TREKK network. TREKK was commonly used by and promoted to other colleagues by nurse and pediatric educators. For nurses, hosting TREKK outreach education sessions established connections to pediatric experts and made obtaining pediatric information and training easier. Among nurses in the rural areas, TREKK was thought to enhance the availability of training events, and TREKK resources were used by other subspecialties in the community, such as primary care and respiratory therapy. Physicians working at multiple sites noted that their TREKK sites had stronger connections to their nearest PERC site, as compared with non-TREKK sites, and others noted that connections to their PERC site for education purposes emerged mainly as a result of TREKK. Network benefits were particularly strong for physicians working in rural areas, supporting local and jurisdictional practice change (physician 2155-BX; 2450-CR), promoting standard of care within emergency medicine and across other subspecialties (physician 2475-KC), and closing knowledge translation gaps, the umbrella term for the multiple activities involved in moving research into practice (physician 2155 BX; 2450-CR). As one physician noted, "being a TREKK site, we're more aware of it, but I find others just aren't aware," suggesting a need to broaden network reach and to promote through multiple channels to ensure that all sites have equal access to pediatric knowledge and training opportunities.

\section{DISCUSSION}

TREKK is the only network in Canada dedicated to providing general ED professionals with current, evidence-based resources and training that is based on stakeholder and user identified priorities ${ }^{12}$ and intended to improve the quality of pediatric emergency care. This study examined health care professionals' information-seeking behaviours and identified ongoing challenges, network successes, and future opportunities for the TREKK network.

To fully comprehend the information seeking of health care professionals, it is important to understand their information needs first. ${ }^{21}$ At its inception, the main goals of TREKK were to determine ${ }^{12}$ and address the knowledge needs of health care professionals working in general EDs by assembling existing evidence and developing usable educational tools through an iterative knowledge translation process ${ }^{22}$ to assist them in the access, adaptation, and implementation of new knowledge. The TREKK needs assessment identified a need to develop tools and interpersonal strategies to fulfill the knowledge needs and preferences of these health care professionals. ${ }^{2}$ In this study, use of resources including the TREKK bottom line recommendations, outreach education sessions, and talking to colleagues in pediatric EDs were common sources of information for both nurses and physicians, suggesting that the priorities and investments of TREKK were in line with the needs of end-users. Locating best-quality evidence throughout the mass of available information can be challenging, ${ }^{21}$ and evidence suggests that resources have to be quickly accessible to be practical at the bedside. $^{23}$ Previous studies have reported a lack of guideline awareness as a barrier to their use in community settings. ${ }^{24}$ To facilitate use, TREKK bottom line recommendations were reviewed at the regional level in some jurisdictions and embedded within local care maps and protocols. Other sites made frequent use of order sets, indicating a more direct strategy to enhance the use of TREKK products. Increasing content visibility and usability, through TREKK promotional materials, desktop icons and apps, and acceptability, and by promotion through local educators, were common strategies used to promote the use of best practice. Findings suggest that network resources and processes are being used as regional benchmarks across a number of sites, but there remain many EDs outside the network who stand to benefit from TREKK resources, which are publicly available.

A salient theme emerging from the data was a remaining level of health care provider discomfort with pediatric cases, suggesting a need for ongoing strategies to mitigate discomfort. This finding is consistent with existing research that supports an overall sense of unease with critical pediatric conditions in the community setting. ${ }^{24-26} \mathrm{~A}$ desire for more frequent exposure to hands-on experiential learning has been previously reported in the community urgent care setting $^{12,24}$ and was reinforced in this study. Discomfort, both in dealing with pediatric cases and in seeking out advice from pediatric experts, was commonly discussed. At a practical level, this discomfort occurred because of infrequent experience with critically ill pediatric patients and was enhanced by exposure to pediatric information, resource sharing, training, and 
contact with pediatric experts. As network connections strengthened over time, health care professionals not only felt an increased comfort level to seek out clinical advice from PERC sites but also commonly shared knowledge gained through these encounters and other offsite training with colleagues in their local ED, amplifying the sharing of knowledge. These results demonstrate that network structure and processes minimized geographic boundaries and provided new opportunities to seek and disseminate knowledge.

The TREKK experience is consistent with the knowledge translation literature, which suggests that a multi-pronged active engagement strategy with research end-users is more likely to result in the uptake and use of knowledge. ${ }^{27,28}$ Elements that have been previously described to improve the effectiveness of knowledge translation interventions include increased exposure, access to and engagement with content experts, and tailoring interventions to the needs and contexts of end-users. ${ }^{29}$ Successful knowledge translation requires understanding and attending to the multidimensional barriers and facilitators that influence knowledge-to-practice gaps. ${ }^{27,30}$ However, barriers are dynamic, reinforcing the importance of evaluation over time to ensure that network strategies continue to be appropriate and relevant to end-users. ${ }^{28}$

\section{Implications}

By gaining a better understanding of network processes from the perspective of front-line health care professionals, this study illuminates current informationseeking practices, a distinct culture in pediatric emergency medicine, and its potential impact on standardizing care across sites. This exploratory study highlights the need to strengthen current efforts through dedicated nurse liaison roles and to expand network reach to include additional sites and specialties such as family medicine. Although network structure and processes were associated with inherent benefits, ongoing challenges suggest a need for continued growth of TREKK activities and additional resources to enhance strategies to improve comfort with pediatric care in general ED settings.

\section{LIMITATIONS}

Our sampling technique aimed to enhance the external validity of our findings by sampling across a range of geographical areas, professions, and previously connected and disconnected sites. Despite our efforts, the majority of study participants held multiple roles, including those as educators or leaders, potentially weighting the results in favour of a more connected subset of health care professionals. It is critical to remember that our study was conducted within the TREKK network, a network in which health care professionals have access to more pediatric-specific resources and training opportunities. As a result, our findings may not have broad transferability to all general EDs without similar resources. Yet, the interview questions sought to identify challenges and opportunities in pediatric emergency medicine generally, rather than focus on network benefits. To further delineate the power of the network, future studies should replicate these interviews with staff working in general EDs outside the network. Future follow-up studies within the network have the potential to identify changes in the network structure and processes over time and to adapt knowledge mobilization efforts accordingly.

\section{CONCLUSIONS}

By gaining a better understanding of network processes from the perspective of front-line health care professionals, this study illuminates current informationseeking practices, a distinct culture in pediatric emergency medicine, and its potential impact on standardizing care across sites. It also highlights the need to strengthen current efforts through dedicated nurse liaison roles and to expand network reach to include additional sites and specialties. Our results reinforce the critical importance of ongoing network evaluation to ensure that network strategies continue to be appropriate and relevant to end-users.

Acknowledgements: The authors acknowledge the ongoing contributions of TREKK network members to network activities, including the Board of Directors, Knowledge Mobilization Advisory Committee, Parent Advisory Committees, PERC Representatives and Coordinators, TREKK Representatives, TREKK Steering Committee, and the TREKK Administrative Centre. LKC, LK, JC, and CL contributed to the study design, and $\mathrm{LKC}$ organized and conducted the interviews. LKC and GB analyzed the transcripts, and $\mathrm{LKC}, \mathrm{GB}$, and CL were involved in theme development and interpretation. LKC composed the manuscript, and TPK, LK, JC, CL, SS, LH, and MJ provided critical input to and revised the manuscript. All authors have read and approved the final manuscript. 
Competing interests: None declared. TREKK is funded by the Government of Canada's Networks of Centres of Excellence Knowledge Mobilization initiative and gratefully acknowledges support from our host institution, the University of Manitoba, as well as support from the Children's Hospital of Eastern Ontario Research Institute, Children's Hospital Foundation of Manitoba, Children's Hospital Research Institute of Manitoba, PERC, Research Manitoba, Stollery Children's Hospital Foundation, University of Alberta, and the Women and Children's Health Research Institute.

\section{REFERENCES}

1. Lang ES, Wyer PC, Haynes RB. Knowledge translation: closing the evidence-to-practice gap. Ann Emerg Med 2007;49(3):355-63.

2. Curran J, Abidi SS. Evaluation of a discussion forum for knowledge sharing among emergency practitioners: a social network approach. Stud Health Technol Inform 2006;124:941-6.

3. Upper Midwest Rural Health Research Centre. Rural emergency department staffing: Potential implications for the quality of emergency care provided in rural areas; 2007. Available at: http://rhrc.umn.edu/wp-content/files_mf/case yedstaffing.pdf.

4. Canadian Institute for Health Information. Understanding emergency departments and children in Ontario; 2008. Available at: https://secure.cihi.ca/free_products/aib_apr24_ 08_en.pdf.

5. Knapp JF, Simon SD, Sharma V. Quality of care for common pediatric respiratory illnesses in United States emergency departments: analysis of 2005 National Hospital Ambulatory Medical Care Survey Data. Pediatrics 2008; 122(6):1165-70.

6. Hampers LC, Faries SG. Practice variation in the emergency management of croup. Pediatrics 2002;109(3):505-8.

7. Johnson DW, Craig W, Brant R, et al. A cluster randomized controlled trial comparing three methods of disseminating practice guidelines for children with croup [ISRCTN73394937]. Implement Sci 2006;1(1):10.

8. Freedman SB, Gouin S, Bhatt M, et al. Prospective assessment of practice pattern variations in the treatment of pediatric gastroenteritis. Pediatrics 2011;127(2):e287-95.

9. Fitzgerald L, Harvey G.. Translational networks in healthcare? Evidence on the design and initiation of organizational networks for knowledge mobilization. Soc Sci Med 2015;138:192-200

10. The Health Foundation. Leading networks in healthcare: Learning about what works - the theory and the practice; 2013. Available at: http://www.health.org.uk/publication/ leading-networks-healthcare.

11. Borgatti SP, Foster PC. The network paradigm in organizational research: a review and typology. 7 Manage 2003; 29(6):991-1013.

12. Scott SD, Albrecht L, Given LM, et al. Pediatric information seeking behaviour, information needs, and information preferences of health care professionals in general emergency departments: Results from the Translating Emergency Knowledge for Kids (TREKK) Needs Assessment. CFEM 2018;20(1):89-99.
13. Curran JA, Murphy AL, Abidi SS, Sinclair D, McGrath PJ. Bridging the gap: knowledge seeking and sharing in a virtual community of emergency practice. Eval Health Prof 2009; 32(3):312-25.

14. Dawes M, Sampson U. Knowledge management in clinical practice: a systematic review of information seeking behavior in physicians. Int 7 Med Inform 2003;71(1):9-15.

15. Provan KG, Kenis P. Modes of Network Governance: Structure, Management, and Effectiveness. 7 Public Adm Res Theory 2008;18(2):229-52.

16. Wolfe AW. Social network analysis: methods and applications. Am Ethnologist 1997;24(1):219-0.

17. Kelman I, Luthe T, Wyss R, et al. Social Network Analysis and Qualitative Interviews for Assessing Geographic Characteristics of Tourism Business Networks. PLoS One 2016;11(6):e0156028.

18. Williams TA, Shepherd DA. Mixed method social network analysis. Organ Res Methods 2016;20(2):268-98.

19. Klassen TP, Curran J, Ripstein J, et al. eds. Translating Emergency Knowledge for Kids (TREKK): A social networking analysis of a national pediatric emergency network. Presented at 11th Annual Child Health Research Day; 2016 October 1; Winnipeg, MB.

20. Hsieh HF, Shannon SE. Three approaches to qualitative content analysis. Qual Health Res 2005;15(9):1277-88.

21. Davies K, Harrison J. The information-seeking behaviour of doctors: a review of the evidence. Health Info Libr 7 2007; 24(2):78-94.

22. Featherstone RM, Leggett C, Knisley L, et al. Creation of an Integrated Knowledge Translation Process to Improve Pediatric Emergency Care in Canada. Health Commun 2017 May 1-8.

23. Sackett DL, Straus SE. Finding and applying evidence during clinical rounds: the "evidence cart". $7 A M A$ 1998; 280(15):1336-8.

24. Gilleland J, McGugan J, Brooks S, Dobbins M, Ploeg J. Caring for critically ill children in the community: a needs assessment. BM7 Qual Saf 2014;23(6):490-8.

25. Simon HK, Sullivan F. Confidence in performance of pediatric emergency medicine procedures by community emergency practitioners. Pediatr Emerg Care 1996;12(5):336-9.

26. Falgiani T, Kennedy C, Jahnke S. Exploration of the barriers and education needs of non-pediatric hospital emergency department providers in pediatric trauma care. Int 7 Clin Med 2014;5(2):56-62.

27. Grol R, Grimshaw J. From best evidence to best practice: effective implementation of change in patients' care. Lancet 2003;362(9391):1225-30.

28. Lau R, Stevenson F, Ong BN, et al. Effectiveness of strategies to facilitate uptake or implementation of complex interventions: A systematic review of reviews. Implement Sci 2015;10(1):A67.

29. LaRocca R, Yost J, Dobbins M, Ciliska D, Butt M. The effectiveness of knowledge translation strategies used in public health: a systematic review. BMC Public Health 2012;12(1):751.

30. Grimshaw JM, Eccles MP, Lavis JN, Hill SJ, Squires JE. Knowledge translation of research findings. Implement Sci 2012;7(1):50. 


\section{APPENDIX}

\section{Semi-structured interview guide}

1. Tell me about your role in the ED. Do you work in any other EDs, health centres, wards, or clinics?

2. Where is your nearest children's hospital? Is this your first point of contact for seeking guidance/ updates/information on pediatric emergency medicine?
3. Broadly speaking, what factors do you see impacting your ability to seek pediatric emergency information or training?

4. Do you seek advice/information from anywhere else (other hospitals, institutions, or organizations)?

5. Can you think of any strategies/initiatives that could help your ED better connect to your nearest children's hospital or tell me about any that have worked well to date? 